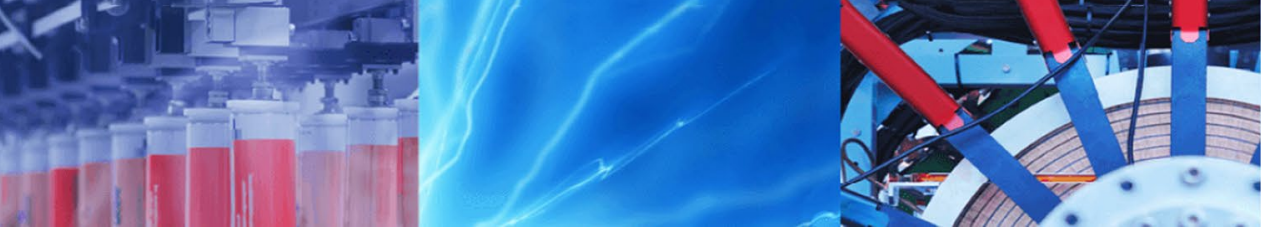

Research Article

\title{
Application to nonlinear mechanical systems with dry friction: hard bifurcation in SD oscillator
}

\author{
P. Ndy Von Kluge ${ }^{1}$ (1) G. Djuidjé Kenmoé ${ }^{1}$.T. C. Kofané ${ }^{1}$
}

(c) Springer Nature Switzerland AG 2019

\begin{abstract}
The stochastic model approach of a nonlinear non-smooth dynamical system with the probable occurrence of stochastic P-bifurcations is devoted. The response probability density functions (PDF) for the stationary measure of a smooth and discontinuous oscillator under moving loads belt frictions is constructed. The appearance of abrupt changes and unpredictable events illustrate the complexity of the system. The stationary measure varies continuously with system's parameters and describes various kinds of catastrophic events. In light of these facts, the behaviour of the "stochastic attractors" is examined through the stationary solution of the PDF. According to Zeeman, in the phenomenological approach in the presence of noise, "a change in character of the density function as a parameter is varied is known as p- bifurcation". Numerous new events unique to non-smooth systems are observed under slight variation of system's parameters. Discontinuous bifurcations are defined as the "hard bifurcations" that were the subject of Catastrophe theory. Peaks numbers increase as coefficient of friction $\mu$ (or smoothness parameter $a$ ) increases. Numerical simulations are presented that provide insights into the dynamics of these oscillators.
\end{abstract}

Keywords Hard bifurcation · Stochastic attractor · Stochastic bifurcation · Self-excited SD oscillator

\section{Introduction}

Discrete and instantaneous transition are always observed in physical systems. Very often in engineering and biology, vibrations are influenced by physical discontinuities. The stick-slip phenomenon occurs.

Systems with friction as the state variables representing the system dynamics are confined to both subspaces at different instants of time belongs to the category of "discontinuous systems". It should be noted that, because of discontinuous vector fields, we will focus on Filippov systems. Therefore, stick-slip phenomena are important examples of this last one. Some bifurcations referred as non-smooth bifurcations or C-bifurcations may occur.
Under the effects of periodic impulse and random force; the nonlinear system has some propensities to oscillate.

Complex behaviours occur in the system's response including the transitions among the multiple motion patterns [1], chaotic phenomena [2] and bifurcation Phenomenon, such as phenomenological [3]. Under the action of fast vibration friction properties change. There is the source of "self-sustained Oscillations" under certain conditions. A lot of researches have been done in this field [4]. They are illustrated by a common phenomenon: switching behaviour upon appearance of intermittent events.

Systems with dry friction under necessary conditions show the catastrophic case ("grazing-sliding bifurcation").

Non-smooth systems are receiving a great curiosity because of their ubiquity in applications of biological

This paper was recommended for publication in revised form by Associate Editor 000000.

P. Ndy Von Kluge, pvonkluge@yahoo.fr |'Department of Physics, Laboratory of Mechanics, Materials and Structures, Faculty of Sciences, University of Yaoundé1, P.O. Box 812, Yaoundé, Cameroon.

SN Applied Sciences (2019) 1:1140 | https://doi.org/10.1007/s42452-019-0987-1

Received: 6 February 2019 / Accepted: 25 July 2019 / Published online: 3 September 2019

SN Applied Sciences

A SPRINGer NATURE journal 
and engineering nature. From interaction with the environment, discontinuities occur naturally. Little attention has been paid on bifurcation behaviours of the nonlinear friction system characterized by geometric nonlinearities. Mechanical systems are subject to background vibrations and other sources of noise [5]: The possible structural changes of the asymptotic behaviour of the system under parameter variation. The stick-slip phenomenon and the related intermittent motion are the motivation of our studies, being the key driver of our non- smooth stochastic model.

For studying noise phenomena in nonlinear dynamical systems coupled to a fluctuating environment one of the main statistical characteristics of these systems is the probability density of the solution. These have attracted a growing interest also from a theoretical point of view (see for instance [6, 7]). A whole new area of research is opened, surely not completely known when adding noise to the non-smooth dynamic system. The interplay of dry friction and random forces in terms of so-called P-bifurcations has been reported in [8]. Phenomenological bifurcations are defined here as changes in the distribution laws i.e. "the qualitative changes of the stationary probability distribution of amplitude"; alternatively, it is a sudden change in character of a "stochastic attractor" when the bifurcation parameter of the system passes through a critical value. Under certain circumstances the Collision between the "stochastic attractor" with a "stochastic saddle" created the loss of the stability of the system. It can happen that a slight variation in a parameter can have significant impact on the solution. By "hard bifurcation", we are talking about the abrupt and unpredicted change in the probability density function (PDF), the presence of discontinuities on the support of a stationary measure or PDF of the system.

In 1973, Thompson and Hunt proposed a simple shallow "arch model" to study the buckling behaviour under a static load [9]. Cao et al. [10-12] proposed in 2006 the "smooth and discontinuous" (SD) oscillator where the nonlinearity is irrational. The geometrical nonlinearity caused by large deformation was illustrated. Complex motions and coexisting attractors are also devoted. An applied force moves an object and created the sliding process, otherwise the object sticks. Remove this constraint, the system becomes two dimensional. Consequently, another friction force must be added to separate permanent sliding motions from jumping effects.

Friction constitutes an important area of many other disciplines rich in interesting examples and applications such as seismology and tectonic fault dynamics under geometrical nonlinearities by considering the large scale displacement of the system $[13,14]$ or climate and weather changes with possible stick slip motion [15], Reliability and braking power $[16,17]$ are some illustrations.
Recent studies have successively explained novel behaviour that may occur in such systems $[18,19]$. But, little is known when adding random parameters to "discontinuous systems" in spite of some new publications $[20,21]$.

To avoid the lack of detailed of the statistical characteristic of a random dynamical system, an appropriate choice is to describe the stationary measure. Because of the long term behaviour of solutions with an emphasis of probability densities when talking about the stationary and dynamical behaviour of the system.

In real systems moreover, parameter uncertainty, background vibrations and other sources of noise are ubiquitous.

The dynamic of systems with dry friction subjected to random forces have abrupt changes occurrence when a stable equilibrium is lost. A Gaussian white noise is sufficed to describe the influence of random perturbations.

Terminology from "catastrophe" returned to singularities, discontinuous bifurcations. What types of long-term dynamical behaviour are possible talking about SD oscillator? The "hard bifurcations" were the subject of "Tom's Catastrophe Theory", this proved to be basic to nonlinear friction system with geometric nonlinearity in mechanical engineering. Thus, one cannot help thinking about unexpected events that cannot be predicted for appearance of $p$-bifurcations or how does the structure of the steady state solution set change as the parameters are varied? How to predict (in the presence of noise) qualitative changes in system's behaviour (stochastic perturbations) occurring at these equilibrium points?

The paper is organized into the following sections. Section 2 contains the model studied. Section 3 presents the dry-friction oscillator as a Filippov system. Section 4, is devoted to theoretical discussion and physical phenomena analyse. The conclusion is made in Sect. 5.

\section{System description and modelling assumptions for stochastically perturbed sliding motion}

Consider a non-deformable moving belt, moving with a constant velocity $V_{1}$, the block of mass $m_{1}$ moving in the lying flat surface and connected to a damping capacity $C$ (or damping function $\Phi\left(\mathrm{x}_{1}(\mathrm{t}) ; \mu\right)$ ) and a fixed backing by an inclined linear spring of stiffness coefficient $K$, which is capable of opposing both tension and compression (see Fig. 1a). The block can either ride on the belt, with zero relative velocity with respect to it, or slip on it because friction is added as a constraint on a rough surface between the mass and the belt. We then suppose large displacements of the mass same as large deformations in continuum mechanics so that the 
(a)

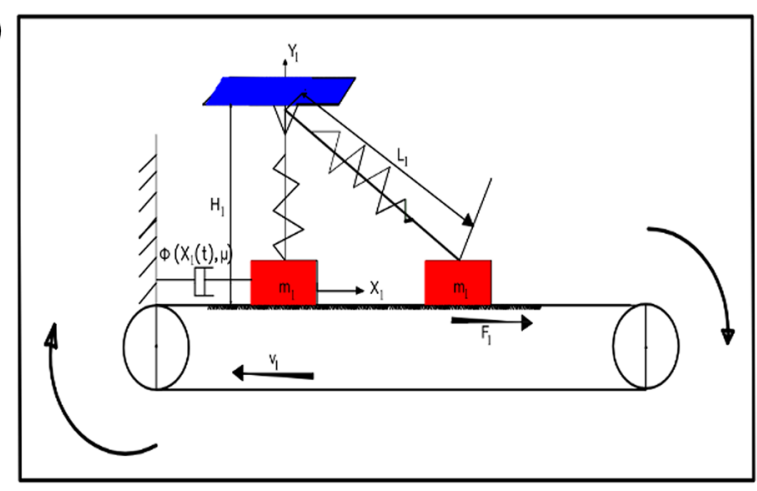

(b)

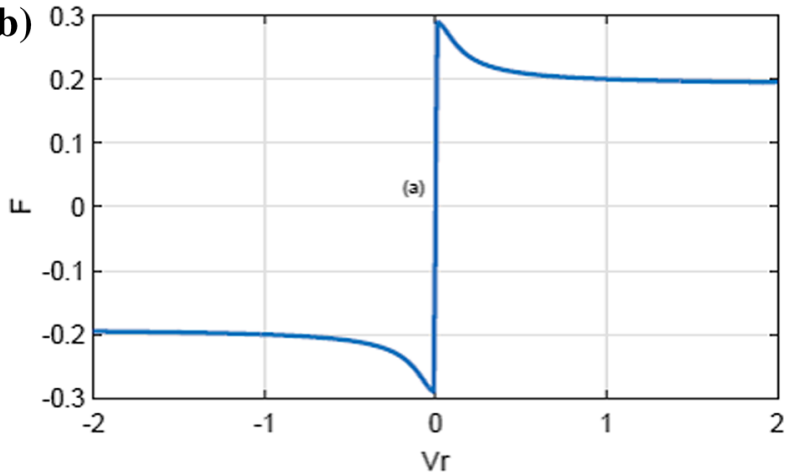

Fig. 1 The mechanical model: a the "self-excited SD oscillator", b Coulomb friction Eq. 2 with $\mathrm{G}_{\mathrm{s}}=\mu\left(\left(\mathrm{g}_{1}-\alpha\left(1-\frac{1}{\sqrt{x^{2}(t)+\alpha^{2}}}\right)\right)+\mathrm{C}_{1}|x|+\mathrm{C}_{2}|\dot{x}|\right)$ $\operatorname{sgn}(\dot{x}) ; \psi \alpha=0.1 ; g_{1}=2.0 ; \mu=0.1 ; \mathrm{v}_{0}=0.0$

system is strongly nonlinear. (Refs. therein). $X_{1}$ is displacement from the rest state. If there is also damping, restoring and external forces, the following equation is studied

$$
m_{1} \frac{d^{2} X_{1}(t)}{d t^{2}}+C \frac{d X_{1}(t)}{d t}+\mathrm{KX}_{1}(\mathrm{t})\left(1-\frac{L}{\sqrt{X_{1}^{2}(t)+L_{1}^{2}}}\right)+\mathrm{F}_{\mu}
$$$$
=\mathrm{F}_{\mathrm{e}} \cos (\mathrm{wt})+\xi(\mathrm{t})
$$

$L_{1}$ and $H_{1}$ are respectively the spring's length and the distance between fixed point and belt, $F_{\mu}$ due to contact friction force. $\xi(t)$ is the normalized source of Gaussian white noise:

$\left\langle\xi(t) \xi\left(t^{\prime}\right)=2 D \delta\left(t-t^{\prime}\right)\right\rangle$,

$\langle\xi(t)=0\rangle$ and

$D$-the noise intensity. The dry friction [22] force $F_{\mu}=G_{s}$ is due to the surfaces in contact and can be defined during the slip mode. The extended friction law is [23]:

$\mathrm{F}_{\mu}=-\mu\left(\mathrm{C}_{0}+\mathrm{C}_{1}\left|\mathrm{x}_{1}\right|+\mathrm{C}_{2}\left|\dot{\boldsymbol{x}}_{1}\right|\right) \operatorname{sgn}\left(\dot{\boldsymbol{x}}_{1}\right)$

So that $x_{1}$ and $\dot{x}_{1}$ are respectively the sliding displacement, and correspondent velocity, $C_{0}$ is the vertical component of the spring force, coefficients $C_{1}$ and $C_{2}$ are respectively the friction interface amplitude, with the correspondent velocity. $\mu$ stands to friction coefficient (equivalent to the static friction coefficient in general). Suppose the weight, $\mathrm{F}_{\mathrm{N}}=C_{0}$ is in the sense of the weight in the contact area assuming that the direction of $C_{0}$ always points down $\left(F_{N}>0\right)$ only if $\mathrm{Mg}>\mathrm{KH}_{1}$, i.e., the weight of the system. Hence:

$\boldsymbol{F}_{\mu}=\mathrm{Mg}-\mathrm{KH}_{1}\left(1-\frac{\boldsymbol{L}}{\sqrt{\boldsymbol{X}_{1}^{2}(\boldsymbol{t})+\boldsymbol{H}_{1}^{2}}}\right) ;$

$\mu= \begin{cases}\mu_{k}, & \frac{d x}{d t}=v_{0} \\ \mu_{s}, & \frac{d x}{d t} \neq v_{0}\end{cases}$

$\mu$ is depending on $v_{r}=\dot{x}-v_{0}$, i.e., the vertical component of the spring force. The friction force $F_{\mu}=G_{s}$ modelled as Stribeck friction between objects in contact is described as

$\mathrm{G}_{\mathrm{s}}=\mu\left(\mathrm{F}_{\mathrm{N}}+\mathrm{C}_{1}\left|\mathrm{x}_{1}\right|+\mathrm{C}_{2}\left|\dot{x}_{1}\right|\right) \operatorname{sgn}\left(\dot{x}_{1}\right) ;$

and is determined by the material characteristics of the block and the belt. If the moving load acceleration is equal to zero, i.e. $\ddot{x}_{1}=0$ when the load sticks, with the belt velocity $\dot{x}_{1}=v_{1}$, thus the value of the friction force

$\left(G=-\mathrm{KX}_{1}(\mathrm{t})\left(1-\frac{\mathrm{L}}{\sqrt{\mathrm{X}_{1}^{2}(\mathrm{t})+\mathrm{H}_{1}^{2}}}\right) \mathrm{C}_{1}\left|\mathrm{x}_{1}\right|+\mathrm{C}_{2}\left|\dot{x}_{1}\right|\right)$

is confined to the interval $\left(-G_{\max }<G<G_{\max }\right)$. Knowing that

$G \max =\mu\left(M g-K H_{1}\left(1-\frac{1}{\sqrt{X_{1}^{2}(t)+H_{1}^{2}}}\right) C_{1}\left|x_{1}\right|+C_{2}\left|\dot{x}_{1}\right|\right)$,

is the total static friction force derived from the product of the friction coefficient with the normal force. Hence the Stribeck friction force $F_{\mu}$ of the system is illustrated in Fig. 1b. We have described the intermittent behaviour (or the set-valued extension) of the system in the differential inclusion of Filippov type as:

$\operatorname{sgn}\left(x_{1}\right)= \begin{cases}1 & \text { if } x_{1}>0 \\ 0 \in[-1,1] & \text { if } x_{1}=0 \\ -1 & \text { if } x_{1}<0\end{cases}$

The equation of motion Eq. (1) can be normalized using the non-dimensional procedure as follows: $x=\frac{x_{1}}{L_{1}} ; w_{0}^{2}=\frac{K}{m_{1}} ;$ $\mathrm{c}=\Phi\left(\mathrm{x}_{1}(t), \mu\right)=\frac{\mathrm{c}}{m_{1 w_{0}}} ; \tau=\mathrm{w}_{0} \mathrm{t} ; \alpha=\frac{H_{1}}{L_{1}} ; v_{0}=\frac{v_{1}}{L_{1}} ; g_{1}=\frac{g}{L_{1} w_{0}^{2}}$. Then, substituting these variables into Eq. (1) we have: 


$$
\begin{aligned}
\ddot{x}+ & c \dot{x}+x\left(1-\frac{1}{\sqrt{x^{2}+\alpha^{2}}}\right) \\
& -\mu\left(C_{0}+C_{1}|x|+C_{2}|\dot{x}|\right) \operatorname{sgn}(\dot{x})=a_{m} \cos (\mathrm{wt})+\xi(\mathrm{t}),
\end{aligned}
$$

where

$C_{0}=\left(g_{1}-\alpha\left(1-\frac{1}{\sqrt{x^{2}(t)+\alpha^{2}}}\right)\right)$

and $(\cdot)$ represents the non-dimensional ratio of time $\tau$. Knowing that $c=\Phi\left(x_{1}(t) ; \mu\right)$. The slip and stick modes characterized the motion of the mass.

$(\mathrm{F} \mu)$ : depends of the velocity $\dot{x}$, and displacement from the rest state $x$. The smoothness parameter $a$. When $a>0$, the system is continuous and for $a=0$, the system nonlinearity is discontinuous. The dynamics of the SD oscillator has been investigated random domains.

The discontinuous case equation of motion of the (SD) Oscillator is given by:

$\ddot{x}+c \dot{x}+(x-z \operatorname{sgn}(x))-\mathrm{d} \operatorname{sgn}(\dot{x})=a_{m} \cos (\mathrm{wt})+\xi(\mathrm{t})$, will be null in practical case. (One between $z$ and $d$ will be zero). Thus, for intermittent mode, "sgn( $x)^{\prime \prime}$ and "sgn $\left(\dot{x}-v_{0}\right)$ ", one could have the excited SD oscillator $(z=d=0)$, the dry friction models $(z=0 ; d=1)$. Filippov [24] representation for the discontinuous SD oscillator is given by: where $d=-\mu\left(C_{0}+C_{1}|x|+C_{2}|\dot{x}|\right)$. At least one coefficient

("The dynamical planar Filippov" system).

A solution of Eq. (7) should be continuously differentiable. If $c=\Phi(x(t) ; \mu)$, this equation describes many physical systems collectively called "SD oscillators".

\section{System with friction}

The stick-slip process occurs by pulling the system i.e. because of the variation of the position.

\subsection{Generic "Filippov system" analysis}

Consider $\mathrm{x} \in \mathrm{R}^{\mathrm{n}}$, and $\mathrm{f}^{(\mathrm{i})}: \mathrm{R}^{\mathrm{n}} \rightarrow \mathrm{R}^{\mathrm{n}}, \mathrm{i}=1,2$, are sufficiently smooth. Let us suppose that the vector field is discontinuous along $\Sigma$,

$x=\left\{\begin{array}{l}L+(x), \text { for } x \in S_{1} \\ L-(x), \text { for } x \in S_{2}\end{array}\right.$

is defined in Fig. 2 (smooth vector fields $L+, L-$ ).

In order to better analyse the non-smooth dynamics, define the intervals as: $\Sigma=\left\{x \in R^{n}, H(x)=0\right\}$, where $H$ is a scalar indicator function

$H_{x}(x)=\frac{\partial H(x)}{\partial x}$

on the a hyper-surface $\Sigma$, (an unique surface of discontinuity: Mathematically, it is the "switching manifold"; physically it corresponds to zero velocity), then the system domain will be divided into three sub-spaces as:

$$
x_{2}-v_{0}<0
$$

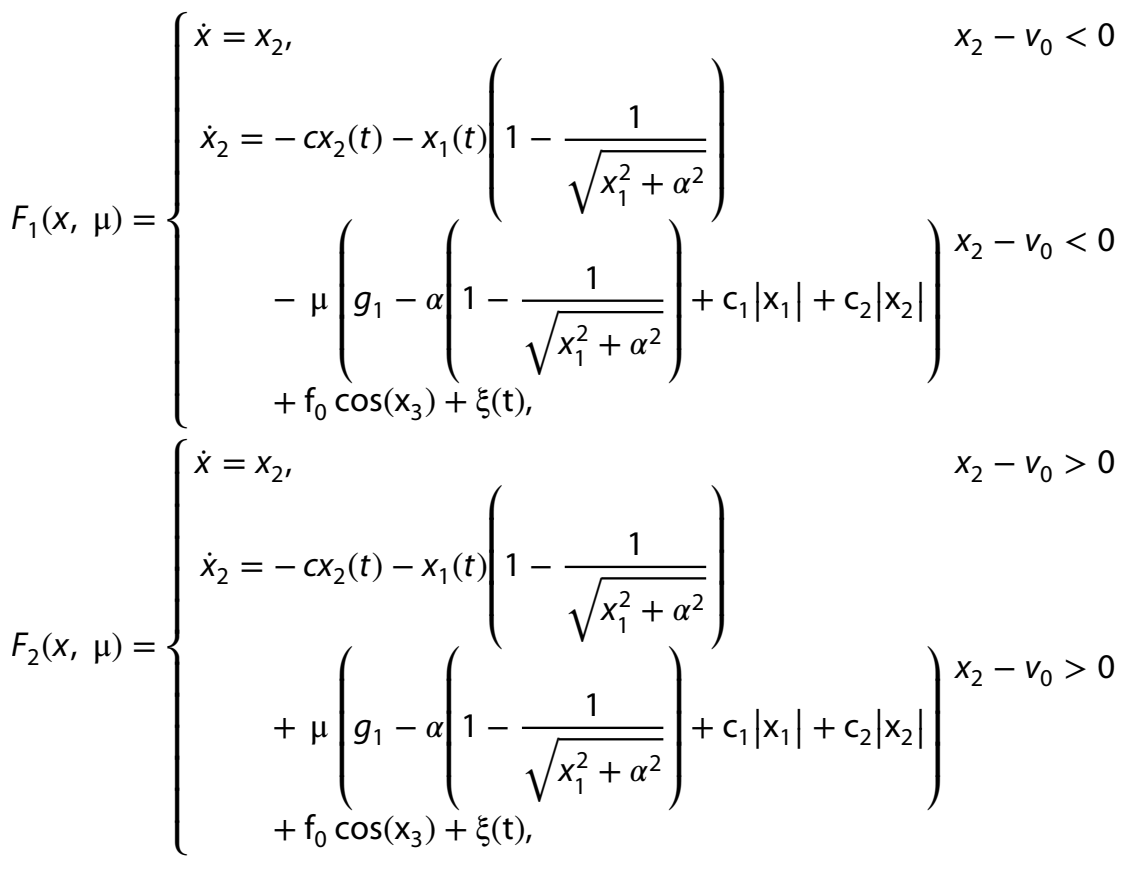


(a)

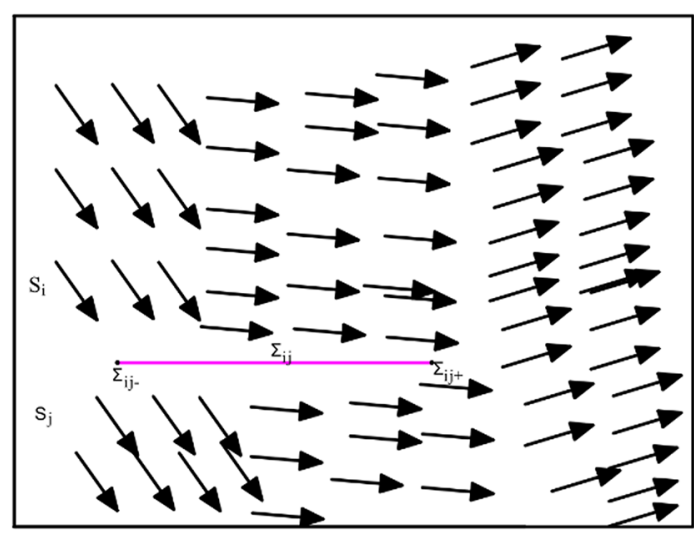

(b)

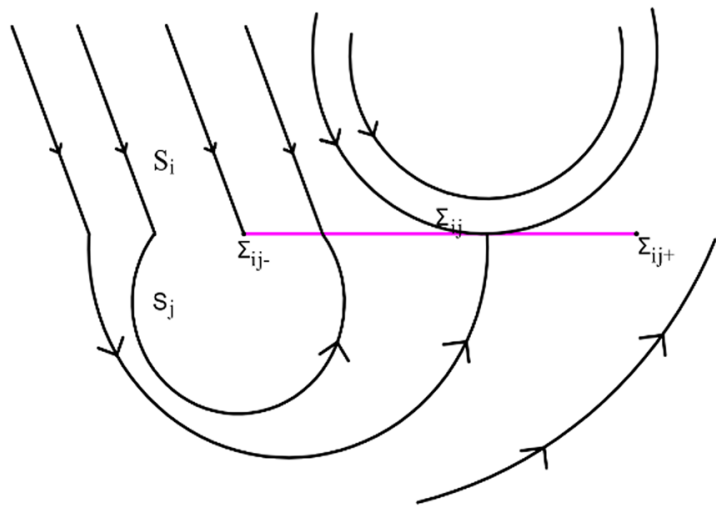

(c)

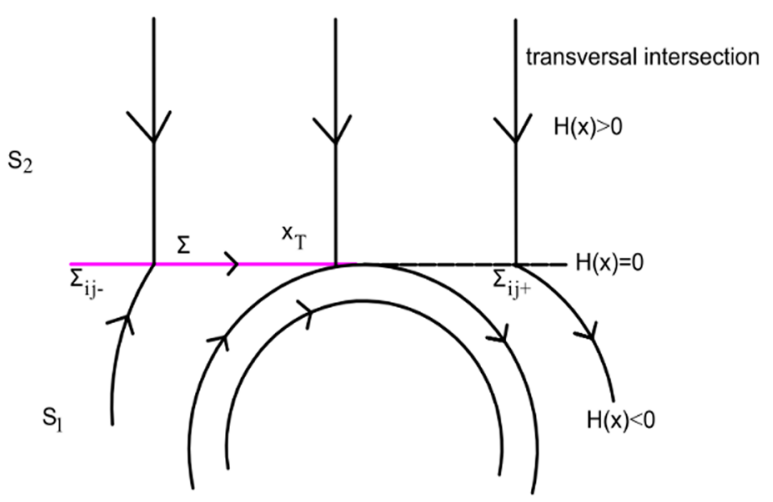

Fig. 2 Filippov representation: a two vector fields $F_{i}$ and $F_{j}$ and an open $\Sigma_{\mathrm{ij}}$ (up), of the corresponding trajectories (down, b). c Filippov' piecewise smooth system

$S_{1}=\left\{x \in R^{n}, H(x)<0\right\} ;$

$S_{2}=\left\{x \in R^{n}, H(x)>0\right\} ;$

$\Sigma=\left\{x \in R^{n}, H(x)=0\right\}$,

The subspaces $S_{1 ; 2}$ and attracting sliding occur in a region on $\Sigma$ obtained with the well-known convexification [25], we can construct the desired general solutions of (8).
Let $\beta_{i j}(x)=\left\langle H_{x}(x), L^{(+)}\right\rangle\left\langle H_{x}(x), L^{(-)}\right\rangle$, be a transversal intersection, $\Sigma$ in which $\langle. .$.$\rangle is the inner product in \mathrm{R}^{n}$. The crossing set $\Sigma_{c} \subset \Sigma$ is defined as $\Sigma_{c}=\left\{x \in \Sigma: \beta_{i j}(x)>0\right\}$ i.e. the total of all points $x \in \Sigma$, the orbit of system (8) at these points crosses the separation area $\Sigma$ i.e., the orbit reaching $x$ from $S_{i}$ concatenates with the orbit entering $S_{j}, i \neq j$, from $x$. the complement to $\Sigma_{c}$ in $\Sigma: \Sigma_{s}=\left\{x \in \Sigma: \beta_{i j}(x) \leq 0\right\}$, where at these points $x \in \Sigma_{s}$, the orbit of system (8) which reaches $x$ does not leave $\Sigma$ and will therefore have to move along $\Sigma$. Since the vector field $H_{x}(x)$ determines the system flow along the barrier of separation, we have $H_{\Sigma}^{\top}(x)=0$, (In the sliding domain, $\mathrm{T}$ is a tangent point at the boundary. Both vectors $\mathrm{H}(\mathrm{i})(\mathrm{T})$ are non-vanishing values, but one of them is tangent to $H_{\Sigma}^{\top}(x)=(0,1)$ : equilibria of the boundary, and singular sliding points) (See Fig. 2).

\subsection{Numerical solution of "differential equations with switching conditions" in the case of Filippov}

In the piecewise smooth system (8), $L(x)$ is not well defined when $x$ is on the discontinuity surface $\Sigma$. A way to define the vector field on $\Sigma$ is to consider the Filippov approach that is the set valued extension $\mathrm{G}(\mathrm{x})$ below:

$\dot{x} \in G(x)= \begin{cases}L_{+}(x, \mu), & x \in S_{1} ; \\ C O\{L+(x, \mu), L-(x, \mu)\}, & x \in \Sigma ; \\ L_{-}(x, \mu), & x \in S_{1}\end{cases}$

where $\left(\mathrm{L}_{+}(\mathrm{x}) ; \mathrm{L}_{-}(\mathrm{x})\right)$ are given by the smooth functions, and $\overline{C O}\{A\}$; is a vector field along the barrier of discontinuity, ("the closure of the convex hull"). It denotes the smallest closed convex set containing A.

$\overline{\operatorname{CO}}\left\{\mathrm{L}_{+}, \mathrm{L}_{-}\right\}=\left\{\mathrm{L}_{\mathrm{G}}: x \in \mathrm{R}^{\mathrm{n}} \rightarrow \mathrm{R}^{\mathrm{n}}:\right.$
$\left.\mathrm{L}_{\mathrm{G}}=(1-\alpha) \mathrm{L}_{+}+\alpha \mathrm{L}_{-}, \alpha \in[0,1]\right\} ;$

then the system vector field can be described by a "differential inclusion" (systems with multi valued right-hand sides).

\subsection{The convex approach of non-smooth dynamics}

Talking about the nonlinear system with discontinuous right-hand side

$\dot{x}=L(x)=\left\{\begin{array}{l}L+(x), \text { for } v(x)>0 \\ L-(x), \text { for } v(x)<0\end{array}\right.$

When $\mathrm{x}$ is on hyper-surface $\Sigma$ then $v(x)=0$. In order to study the dynamics in the neighbourhood of the equilibrium set, the endpoints are studied separate from the other points of the equilibrium set $[26,27]$. Mechanical systems with 
set-valued friction, equilibrium sets will occur, generically when a system switches between two systems (suppose the intermittent behaviour of the friction force $G \equiv I)$, then we form their convex combination as

$\dot{x}= \begin{cases}L(x, \lambda)=\frac{l_{+}(x)+I_{-}(x)}{2}+\lambda \frac{l_{+}(x)-I_{-}(x)}{2} & \\ \operatorname{sgn}(v) & (\text { if } v \neq 0) ; \\ {[-1 ;+1]} & (\text { if } v=0) ;\end{cases}$

Thus $\mathrm{L}(\mathrm{x} ;+1) \equiv \mathrm{L}_{+}(\mathrm{x})$ and $\mathrm{L}(\mathrm{x} ;-1) \equiv \mathrm{L}-(\mathrm{x})$. The standard approach then seeks so-called sliding modes which satisfy " $\dot{x}=0$ on $v=0$ ".

\section{Results and discussion}

It is evident that a deep analysing of the role of nonsmooth sliding process of our model is of crucial importance in the nature and many engineering cases. Friction processes upon geometrical nonlinearity with large deformations open new windows to observe "Tom's catastrophic Theory" in SD oscillator. We have computed Eq. (7) numerically to illustrate the theoretical predictions using fourth-order Runge-Kutta algorithm. In all the calculations we assume that: $c=0.048, x_{\mathrm{fk}}=0.25 ; \mathrm{x}_{\mathrm{fs}}=1.0 ; \mathrm{f}_{0}=0.85$; $a=0.4 ; C_{0} \neq 0 ; C_{1}=C_{2}=0 ; \mu=0.5$. Stick-slip effects in friction oscillators are very complicated. The asymptotic convergence (or divergence) properties of the trajectories of non-smooth systems are observed. Furthermore, we introduced a smooth function which approximates the discontinuous drift (see "Appendix").

The system exhibits different shapes of periodic windows follow by a sudden occurrence of chaotic responses certainly due from jumps that are characteristics of "grazing-sliding bifurcations".

Figure 3 shows the influence of friction coefficient in the dynamic of the system. We have observed the time histories and phase plane plots of the solution when varying friction Coefficients $\mu=0.0 ; 0.1 ; 0.3$. The stick slips friction and limit cycle oscillation (LCO) are really identified in Fig. 3b, c. But, Fig. 3d, e, shows a segment of trajectory crosses the sliding region, enter into the slipping area and joins back the sliding surface. The stick and slip phases are consecutives. Thus, to verify directly the dynamical features of the motion patterns transition we focus at the statistic occurrence of sticking and sliding times, i.e., the distribution of time intervals the load spends in states $v=0$ (sticking events) and $v \neq 0$ (the statistics of the sliding events). The transition value from stick to slip is reached at $\mathrm{G}_{\mathrm{s}}=\mu \mathrm{F}_{\mathrm{N}}$. The slip event finishes for $\dot{x}_{2}=v_{0}$. Besides, we can count the same number of sticks and slips because the system response ends during a slip mode (Fig. 3d, e).
A random sequence alternating stick and slip events occur. When a stick starts, suppose the mass' position is known, and that the base speed is constant in time, the number of time intervals, or the instants at which stick consecutively slip occur can be estimated.

We consider the bifurcations under changes in the driving force and frequency.

From Fig. 4, both periodic and chaotic responses are presented. We observed an abrupt transition from the periodic to chaotic response and, afterwards, from the chaotic to the periodic effect. The presence of "grazing sliding bifurcations" may cause a sudden jump to chaos. It is also found that sliding dramatically change the characteristics of the frequency-response curve. From the smooth to discontinuous regime, periodic windows and chaotic responses are found. The possible structural changes of the asymptotic behaviour of the system under parameter variation, called bifurcations. A slight modification in a parameter value can give rise to a radical change in the system behaviour. When noiseless nonlinear dynamical system exhibits chaos phenomenon, the chaotic attractor is structurally unstable. Whereby, the periodic motions are found and occupy open sets in the parameter space (see Fig. 4c, d, e).

A special chaotic orbit exits for a fixed set of parameters that fills a finite area. It is follow by a series of islands dominated by different chains of fixed points. Therefore, through successive bifurcations where equilibriums and cycles are first links (see Fig. 4d, e) a transition from periodic to chaotic dynamics occurs. The shape and size of "random attractor" and "random saddle" can change under stochastic perturbation.

Figure $5 a, b$ illustrates the influence of friction in the system. With the increasing of noise intensity, Fig. 5a shows a "hard bifurcation" i.e., Tom's catastrophic (due to "grazing-sliding bifurcations") that occur as parameters vary. Abrupt change involves a discontinuity of the steady state at the bifurcation point. The stationary measures provide the possible distributions of traditional trajectories. Their fixed backings are the perspective areas to typical trajectories in the long run. As usual in this case, there can be a lot of possibilities of stationary measure and more radical changes can occur in response to parameter varies. "Tom's Catastrophe" theory sufficed to be the basic to the study of qualitative dynamics. In some disciplines, mathematical models are efficient for the examination of dynamic behaviour of the system being modelled [28]. According to [29], because of the absence of little relationship between the shape variations of stationary probability density function and the random excitation, it is not easy to describe the true change of topological property of a stochastic system simply based on the shape change of stationary 

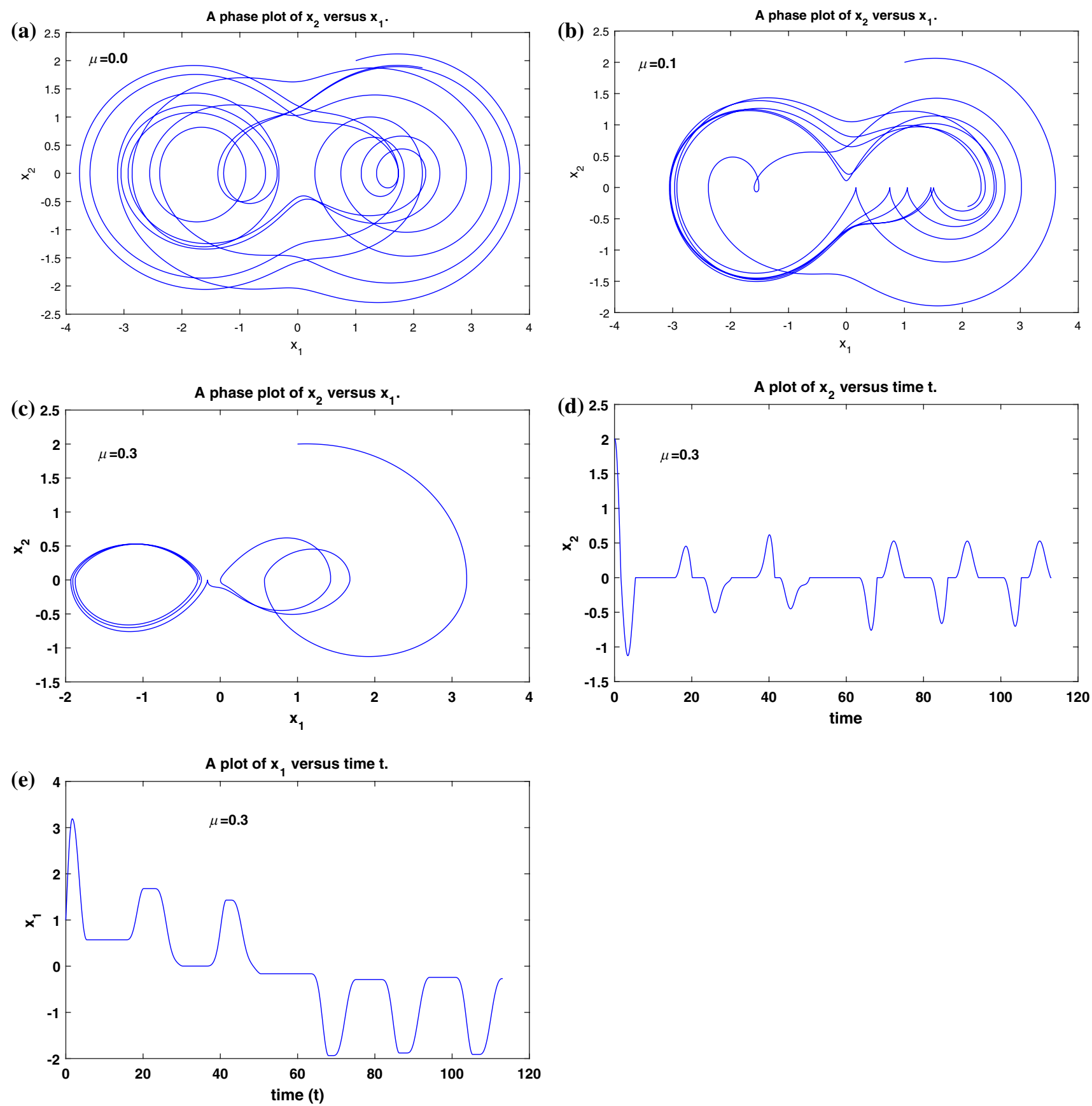

Fig. 3 Sliding Filippov method: $c=0.048 ; a=0.4 ; g_{1}=2.0 ; f_{0}=0.85 ; w=\frac{1}{3}$ : a phase plane plot $\mu=0.0$ no friction, $\mathbf{b}$ phase plane plot with friction, $\mathbf{c}$ limit cycle occurred in phase plane plot as friction increases, $\mathbf{d}-\mathbf{e}^{3}$ dynamics at the transition from stick to slip

PDF. For multiple cycles, noise induces a special type of P-bifurcations.

As the noise intensity (or smoothness parameter) increases, peaks of the probability density function merge and multiplicity of cycle is appeared. While in Fig. $5 b(\mu=0)$, the appearance of $p$-bifurcation diminishes with the increases of noise intensity. In [30], an adjective "hard" is due to a loss of stability of an "invariant set". This phenomenon is verified if the stability involves a discontinuous change; see Fig. 5a, c, e, f. The following changes can be identified in the PDF:

(1) Sliding bifurcations introduce "discontinuous transitions" between different motions. Hence, signatures of the stick-slip transition become dynamically possible above such a critical value. 
(a) 0.5

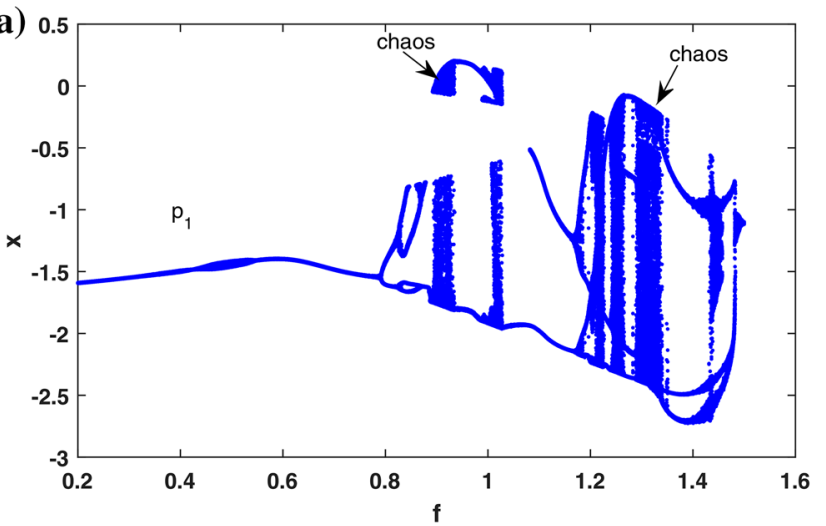

(c)

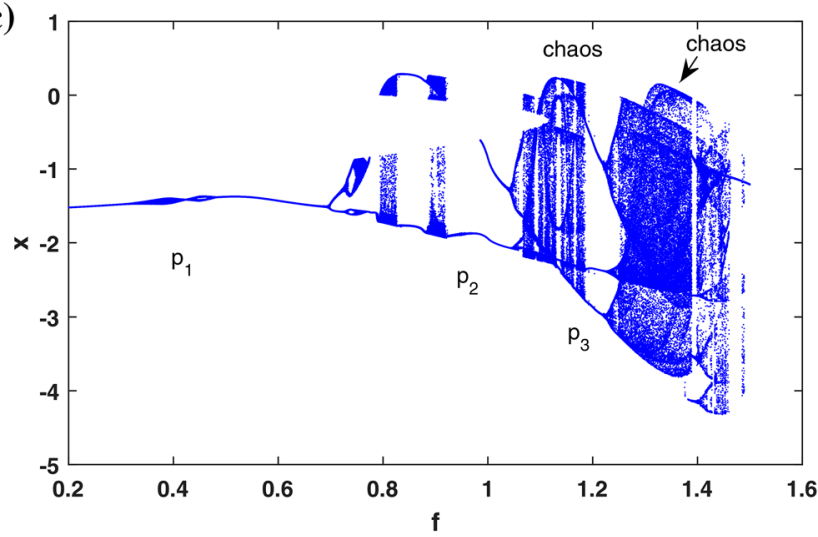

(e)

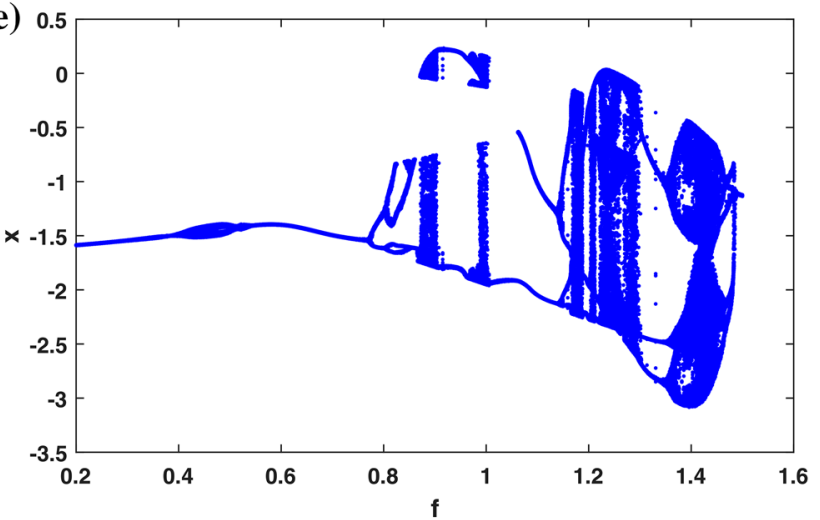

(b)

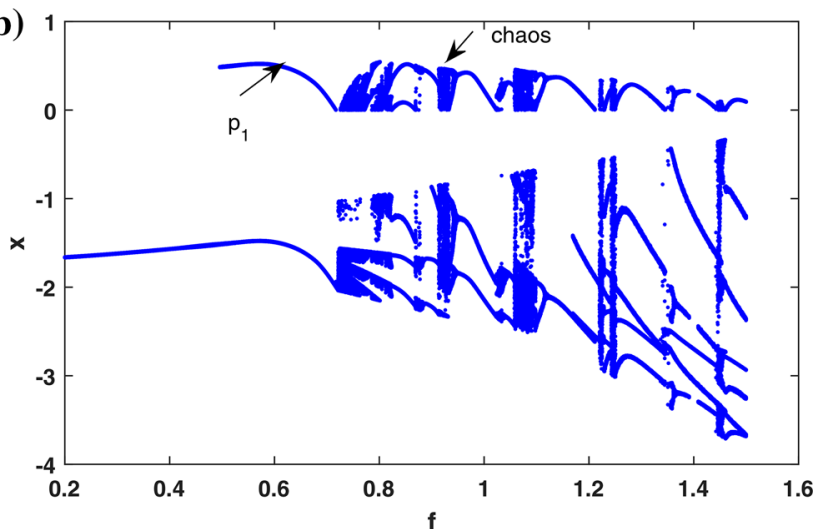

(d)

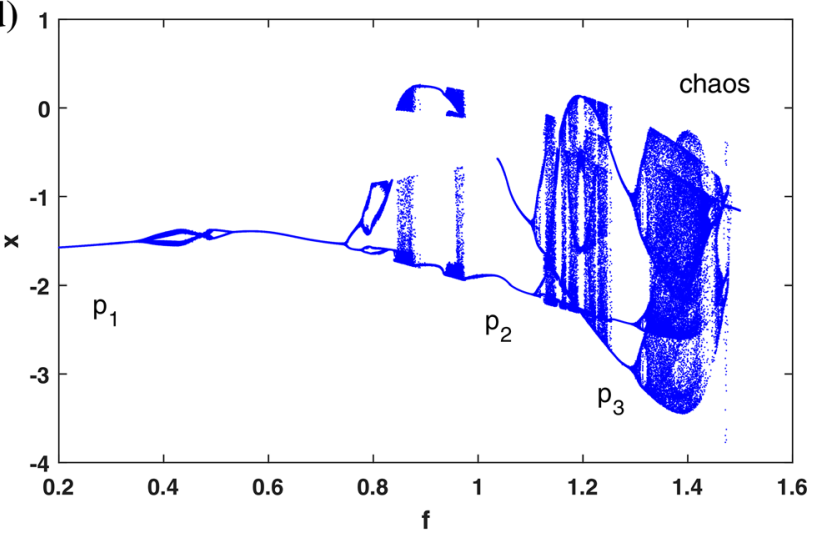

Fig. 4 Bifurcation diagrams for displacement $x$ versus $f_{,}\left(x_{0}=1 ; y_{0}=0\right), c=0.048, x_{f k}=0.25 ; x_{f s}=1.0$ : a $a=0.4, C_{1} \neq 0, C_{2}=C_{3}=0, \mathbf{b} a=0.0, C_{1} \neq 0$, $C_{2}=C_{3}=0 . \mathbf{c} a=0.4, C_{1} \neq 0, C_{2}=0.45, C_{3}=0.5 ; \mathbf{d} \boldsymbol{\alpha}=\mathbf{0 . 4}, C_{1} \neq 0, C_{2}=0.015, C_{3}=0.25 ; \mathbf{e} \mathbf{a}=\mathbf{0 . 4}, C_{1} \neq 0, C_{2}=0.0, C_{3}=0.25$

(2) We are interested in possible changes equilibrium (maxima, minima) provides the asymptotic behaviour for large values of PDF.

(3) Observation of jump effects due to the phenomenon of "grazing-sliding bifurcation".

(4) "Catastrophic (or abrupt) transitions" occur characterized by discontinuous changes in system properties.

Hence, knowing that a system is functioning in one of its asymptotic regimes, is it possible that a slight variation of A parameter triggers a transient toward an abrupt different asymptotic regime? In this case, we say that a "catastrophic transition" occurs.

Figure 5 Shows that with a slight variation of noise to a family of non-smooth system unfolding a bifurcation can lead to a "hard bifurcation" of stationary density functions. We also observe here that, as friction coefficients increase, peaks number, consequently appearing of $p$-bifurcation (see Fig. $5 d-f$ for $\mu=0.1 ; 0.2 ; 0.3$ ). Any parameter can change the behaviour of the system. That's why, Zeeman 

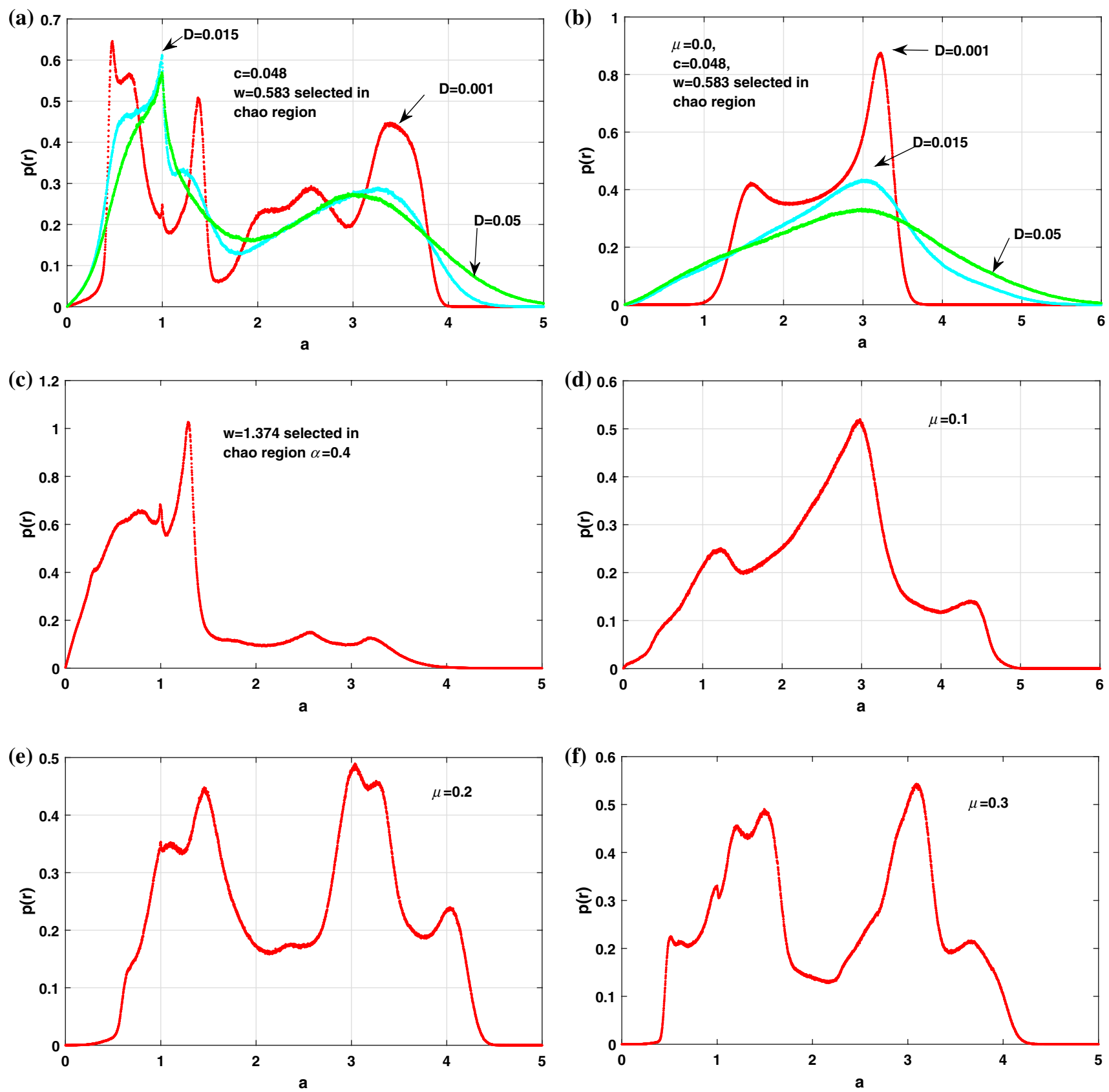

Fig. 5 From Fig. 3a: stationary amplitude distribution for: $D=0.001 ; c=0.048, f_{01}=0.85, C_{1} \neq 0, C_{2}=C_{3}=0, a \quad a=0: 4, b a=0.0, \mathbf{c} a=0.4, \mathbf{d}$ $\mu=0.10$, e $\mu=0.2, \mathbf{f} \mu=0.3$

defined a bifurcation in a stochastic system as a "change in character of the density function as a parameter is varied" $[31,32]$. Hence the phenomenological, or P-bifurcations.

Under parameter change, stationary measures of SD oscillator can illustrate abrupt changes. We observed a change in the number of stationary measures or a discontinuous change in one of their supports (see Fig. 6).

When increasing the damping coefficient c, Fig. $6 \mathrm{a}$ illustrates the increase in term of number of peaks and probability density function (PDF) as above. The same observation is recognized in Fig. $6 \mathrm{~b}$, where the friction coefficients increase with the multiplicity of peaks number and height value of PDF. Another point of view is to cover a deep experimental analysis into the evolutionary behaviour of the "stochastic attractors" in SD oscillator. This may be importantly to understand the stochastic bifurcation in a nonlinear system with noise. In this paper, a "stochastic attractor" is chosen as an invariant for a noisy steadystate response, and the abrupt change of attribution (number, size, attraction) of a stochastic attractor and/or 

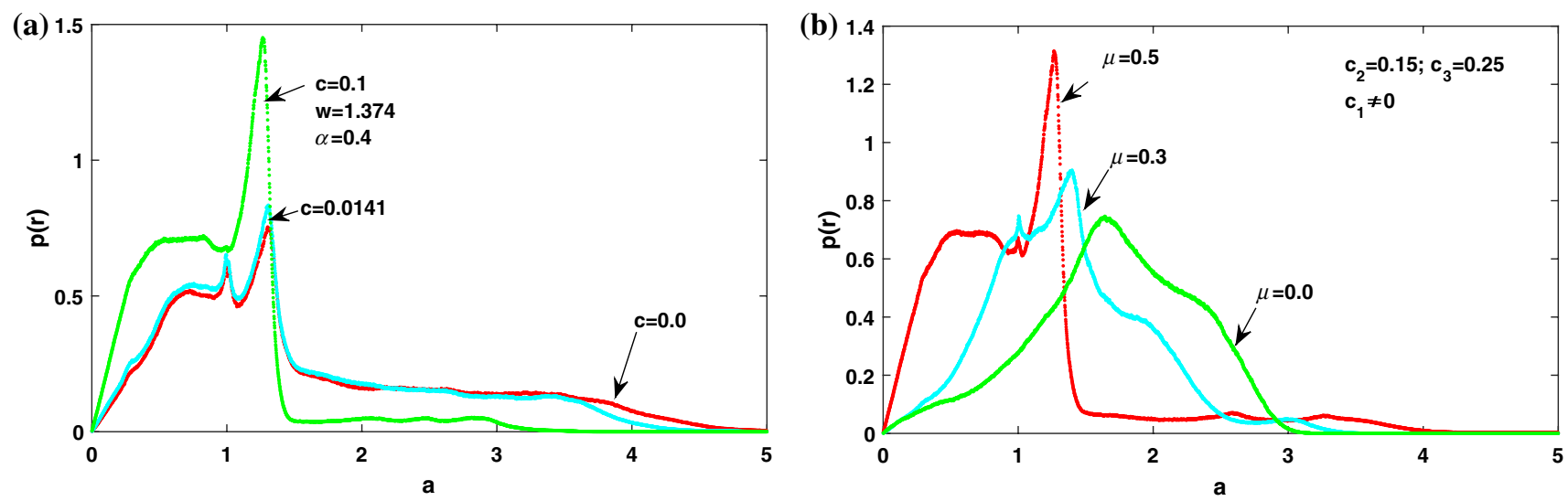

(c)

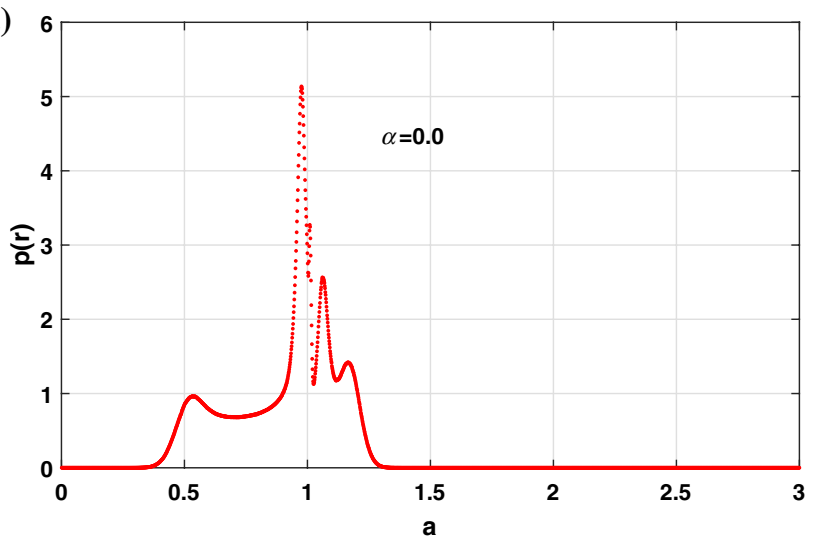

(e) 0.7

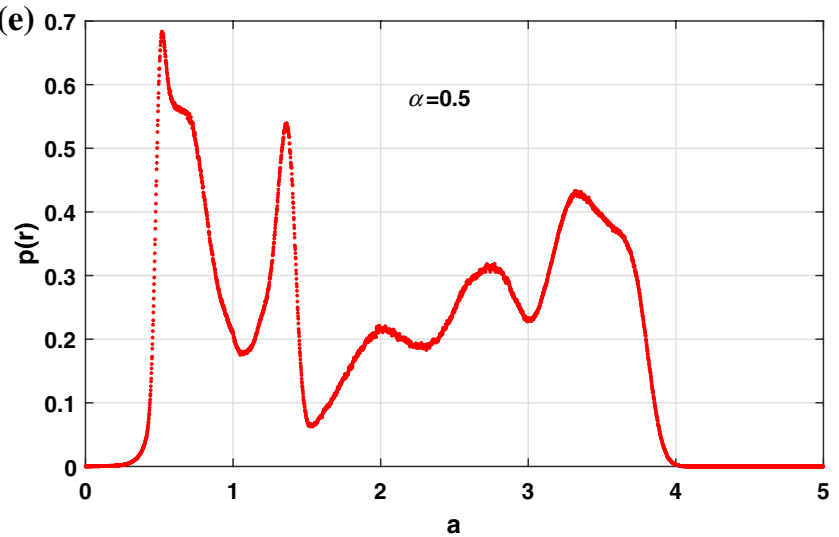

(d)

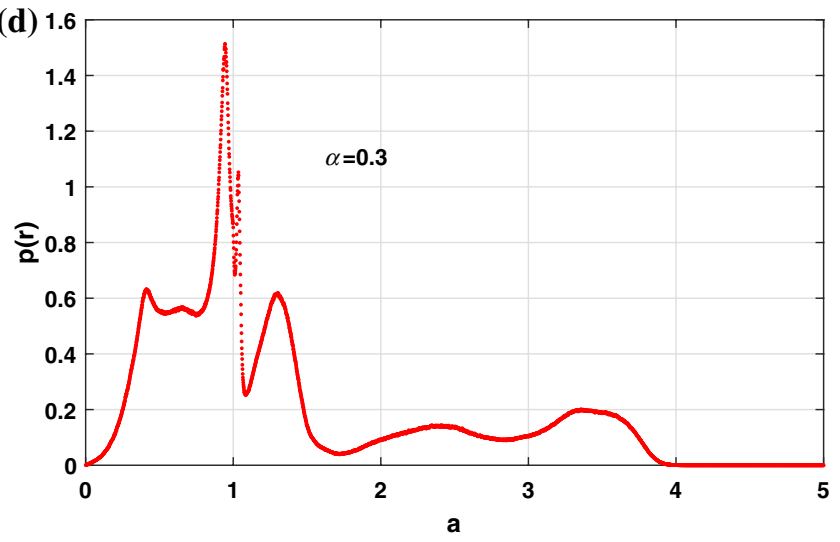

Fig. $6 a=0.4 ; f_{01}=0: 85$ (a) and vary values of damping $c=0.0 ; 0.0141 ; 0.1, C_{1} \neq 0, C_{2}=C_{3}=0$ (b) $C_{1} \neq 0, C_{2}=0.015 ; C_{3}=0.25$ and vary values of friction coefficient $\mu ; \mathbf{c} a=0.0, \mathbf{d} a=0.3 ; \mathbf{e} a=0.5$

a "stochastic saddle" provides a structural change of a stochastic system to demonstrate the bifurcation behaviour.

Figure $6 \mathrm{c}-\mathrm{e}$ show the same observation of increasing of peaks number with the increase of the smoothness parameter $a=0.0 ; 0.3 ; 0.5$. The noiseless system of the SD oscillator undergoes a "pitchfork bifurcation" when the smoothness parameter $a$ increases up to certain value. The stochastic P-bifurcation and the deterministic pitchfork bifurcation in the SD oscillator are related. Suppose $a$ is tending to 0 , the behaviour of the SD oscillator suddenly becomes discontinuous. The speed flow goes through a jump when the system crosses from one side to another in the deterministic system.

This study shows the uncertainties associated with the possible future occurrence of abrupt events, called "catastrophic events". A non-linear approach to detect dynamical transitions and infer the causality behind events (Sliding bifurcation). These Figures try to detect and interpret the existence of multiple equilibriums and can sometimes fail in complex models. Moreover, we highlight promising 
phenomena to detect abrupt effects and to obtain information about the mechanisms behind them.

We can summarize this subsection saying that, the shape of stationary PDF does not depend on the bifurcation parameter [33]. But, the stationary PDF does change its shape at a critical parameter value [34].

\section{Conclusions}

For "discontinuous systems" in Filippov case, the term bifurcation exists in literature with different definitions. They can be inconsistent with one another. The non-uniqueness of solutions is observed. A measure differential inclusion is able to describe discontinuities in the state. We recognize that dry friction is a nonlinearity which is abundant in nature. It presence can induced "self-sustained vibration". The sudden change in a slight variation of any parameter of the system is seems catastrophe, due to loss of stability of the system. This phenomenon occurs in non-smooth systems illustrated in a piecewise-smooth model of a SD oscillator Eq. (7). The slight variation of a parameter has created the "grazing-sliding bifurcations". The occurrence of "catastrophic bifurcation" transition phenomenon is characterized by discontinuous jumps in the equations across a phase space limit.

We were looking to the shape, size and stability of a "stochastic attractor" that may be taken as its character. Whenever the character of an attractor changes radically, there occurs the stochastic bifurcation. P-bifurcation is deemed to occur with a change in the structural behaviour of the probabilistic structure of the state variables.

\section{Appendix}

Due to the lack of continuity, we have construct a function $f_{N}(x)$ which has point wise convergence to the signfunction as $\mathrm{N} \rightarrow \infty$.

$\mathrm{F}_{\mathrm{N}}(\mathrm{x})= \begin{cases}1, & \text { for } \mathrm{x}>\frac{1}{N} \\ \frac{-N^{3} x^{3}}{2}+\frac{3 N x}{2} & \text { for } \frac{-1}{N} \leq \mathrm{x} \leq \frac{1}{N} \\ -1, & \text { for } \mathrm{x}<\frac{1}{N}\end{cases}$

We used $f_{N}(x)$ in order to avoid the discontinuous challenges by the sign function.

\section{Compliance with ethical standards}

Conflict of interest The authors declare that they have no conflict of interest.

\section{References}

1. Rong H, Meng G, Wang X, Xu W, Fang T (2004) Response statistic of strongly non-linear oscillator to combined deterministic and random excitation. Int J Non Linear Mech 39(6):871-878

2. Rajendiran S, Lakshmi P (2016) Simulation of PID and fuzzy logic controller for integrated seat suspension of a quarter car with driver model for different road profiles. J Mech Sci Technol 30(10):4565-4570

3. Huang Z, Zhu W, Suzuki Y (2000) Stochastic averaging of strongly non-linear oscillators under combined harmonic and white-noise excitations. J Sound Vib 238(2):233-256

4. Hinrichs N (1997) Reibungsschwingungen mit Selbst- und Fremderregung. VDIVerl., Duesseldorf

5. Horsthemke W, Lefever R (1984) Noise-induced transitions. Springer, Berlin

6. Tateno T (2002) Noise-induced effects on period-doubling bifurcation for integrate and fire oscillators. Phys Rev E (3) 65(2):021901

7. Arnold L (1998) Random dynamical systems. Springer, Berlin

8. Gulyayev V, Andrusenko E, Glazunov S (2019) Computer simulation of dragging with rotation of a drill string in $3 \mathrm{D}$ inclined tortuous bore hole. SN Appl Sci 1:126. https://doi. org/10.1007/s42452-018-0121-9

9. Li ZX, Cao QJ, Léger A (2016) Complex dynamics of an archetypal self-excited SD oscillator driven by moving belt friction. Chin Phys B 25:010502-1-010502-9

10. Cao QJ, Wiercigroch M, Pavlovskaia EE, Grebogi $C$, Thompson JMT (2006) Archetypal oscillator for smooth and discontinuous dynamics. Phys Rev E 74:046218-1-046218-5

11. Cao QJ, Wiercigroch M, Pavlovskaia EE, Grebogi C, Thompson JMT (2008) The limit case response of the archetypal oscillator for smooth and discontinuous dynamics. Int J Nonlinear Mech 43:462-473

12. Cao QJ, Wiercigroch M, Pavlovskaia EE, Thompson JMT, Grebogi C (2008) Piecewise linear approach to an archetypal oscillator for smooth and discontinuous dynamics. Philos Trans $\mathrm{R}$ Soc A 366:635-652

13. Burridge R, Knopoff $L$ (1967) Model and theoretical seismicity. Bull Seismol Soc Am 57:341-371

14. Carlson JM, Langer JS (1989) Mechanical model of an earthquake fault. Phys Rev A 40:6470-6484

15. Sergienko OV, Macayeal DR, Bindschadler RA (2009) Stick-slip behaviour of ice streams: modelling investigations. Ann Glaciol 50:87-94

16. Li B (2007) Analysis of non-smooth dynamics of brake system with dry-friction. MS thesis, Tianjin University, Tianjin. (in Chinese)

17. Rui-Lan T, Qi-Liang W, Zhong-Jia L, Xin-Wei Y (2012) Dynamic analysis of the smooth-and-discontinuous oscillator under constant excitation. Chin Phys Lett 29(8):084706

18. Cao Qingjie, Léger Alain, Wiercigroch Marian (2017) A smooth and discontinuous oscillator theory, methodology and applications. Springer, Berlin

19. von Kluge PN, Djuidjé Kenmoé G, Kofané TC (2015) Dry friction with various frictions laws: from wave modulated orbit to stick-slip modulated. Mod Mech Eng 5:28-40

20. Simpson DJW, Kuske R (2014) Stochastic perturbations of periodic orbits with sliding. arXiv: 1404.6845v1 [math.DS]

21. Jeffrey MR, Simpson DJW (2013) Non-Filippov dynamics arising from the smoothing of nonsmooth systems, and its robustness to noise. arXiv: 1310.8328v1 [math:DS]

22. Anderson J, Ferri A (1990) Behavior of a single-degree-offreedom-system with a generalized friction law. J Sound Vib 140(2):287-304 
23. Danca MF, Lung N (2013) Parameter switching in a generalized Duffing system: finding the stable attractors. Appl Math Comput 223:101-114

24. Filippov AF (1988) Differential equations with discontinuous right-hand sides. Kluwer Academic Publication, Dortrecht (Russian 1985)

25. Utkin VI (1992) Sliding modes in control and optimization, vol 116. Springer, Berlin

26. Leine RI, Nijmeijer H (2013) Dynamics and bifurcations of nonsmooth mechanical systems, vol 18. Springer, Berlin

27. Santhosh B, Narayanan S, Padmanabhan C (2014) Numericanalytic solutions of the smooth and discontinuous oscillator. Int J Mech Sci 84:102-119

28. Kerschen G, Worden K, Vakakis A, Golinvala J (2006) Past, present and future of nonlinear system identification in structural dynamics. Mech Syst Signal Process 20:505-592

29. Meunier C, Verga AD (1988) Noise and bifurcation. J Stat Phys 50(2):345-375
30. Arnold VI, Afrajmovich VS, Yu S, Il'yashenko Y, Shil'nikov LP (1999) Bifurcation theory and catastrophe theory. Springer, Berlin

31. Zeeman EC (1988) Stability of dynamical systems. Nonlinearity $1(1): 115-155$

32. Zeeman EC (1988) On the classification of dynamical systems. Bull Lond Math Soc 20(6):545-557

33. Baxendale $P$ (1986) Asymptotic behavior of stochastic flows of diffeomorphisms. In: Ito K, Hida T (eds) Stochastic processes and their applications. Lecture notes in mathematics, vol 1203. Springer, Berlin, pp 1-19

34. Crauel H, Flandoli F (1998) Additive noise destroys a pitchfork bifurcation. J Dyn Diff Equ 10:259-274

Publisher's Note Springer Nature remains neutral with regard to jurisdictional claims in published maps and institutional affiliations. 